\title{
Ultrastructure of Human Pancreatic Cancer Cells Treated with a TBK1 Inhibitor
}

\author{
Linda Yasui $^{1 *}$, Nicole Baker ${ }^{2}$, Hannah Savage $^{1}$ and Channing Der ${ }^{2}$ \\ 1. Northern Illinois University, Department of Biological Sciences, DeKalb, IL, USA. \\ 2. University of North Carolina, Department of Pharmacy, Chapel Hill, NC, USA. \\ * Corresponding author: 1yasui@niu.edu
}

Pancreatic ductal adenocarcinoma (PDAC) is a deadly disease having a dismal 5-year survival prognosis. Current therapeutic options are insufficient to stem the disease and most patients die within 1 year of diagnosis. KRAS is an attractive target for drug discovery due to its key role in PDAC initiation and maintenance and it has been extensively studied in the quest to develop novel therapeutic approaches. Moreover, an activating mutation in $K R A S$ is found in more than $95 \%$ of all pancreatic cancer patients. Unfortunately, direct inhibition of KRAS has not proven to be a successfully strategy. Tank-binding kinase 1 (TBK1), a downstream protein kinase component of the less studied K-RAS RALB effector pathway, has been identified as a potential requirement for cancer cell growth driven by K-RAS [1]. As a consequence, TBK1 studies are gaining momentum as a target in the treatment of PDAC [2].

TBK1 is activated downstream of RAS-mediated activation of RALB and the Sec5 component of the exocyst complex to promote tumorigenesis through survival signaling via direct phosphorylation of AKT. On the other hand, RALB also interacts with Exo84 (another component of the exocyst) to promote autophagosome assembly by recruiting beclin to act as a scaffold for autophagy [3]. We hypothesized that inhibition of TBK1 will tilt the equilibrium between tumorigenesis and autophagy towards autophagy, thereby identifying an important signalling component in tumorigenesis of the RASRALB pathway AND potentially identifying a mechanism of cell death by autophagy. Autophagic flux was investigated in a timed study visualizing autophagosomes by transmission electron microscopy (TEM). Appearance of increased numbers or complexity of autophagosomes in TBK1 inhibited cells supports a synthetic lethal cooperation between abnormal KRAS activation and TBK1 inhibition.

Human pancreatic adenocarcinoma (HPAC), a PDAC cell line, was used for this study. Cells were maintained in DMEM medium supplemented with $10 \%$ fetal bovine serum and penicillin/streptomycin in a $37^{\circ} \mathrm{C}$ incubator gassed with $5 \% \mathrm{CO}_{2}$. On the day of the experiment, HPAC cells were treated with 5 $\mu \mathrm{M}$ LSN3090729 in $0.01 \%$ DMSO or sham treated with $0.01 \%$ DMSO for 1, 6, 12 or 24 hours. LSN3090729, a 4-aryl-2-aminopyrimidine derivative, is a selective TBK1 kinase inhibitor as determined by biochemical and cellular analyses [4] that has minimal off-target effects as tested against a panel of over 100 different kinases [3]. At the end of treatment, cells were collected by trypsinization, washed with PBS and then processed for TEM as previously described by us [5]. Thirty images were acquired for each treatment and then analyzed for the cellular polymorphism and cell polarity and for the presence of autophagosomes, vacuoles and dense staining mucin granules. Clathrin coated pits were rarely found suggesting methuosis or cell drinking was not enhanced and did not drive the formation of numerous cellular vacuoles.

Ultrastructural analysis of untreated HPAC cells revealed round or oval-shaped cells containing round or oval-shaped nuclei. Approximately $1 / 3$ of the cell profile was taken up by nuclei. More disperse euchromatin staining was predominantly observed and on average, 1 nucleolus profile was observed per nucleus. Abundant densely staining granules, similar in appearance to mucin granules, were found in 
control cells. Identification of the densely staining granules as mucin granules is supported by the fact that mucin secreting cells are a major cell type from which pancreatic tumors are formed and histochemical studies show secretory granules having the same ultrastructure as observed here, stained positive for mucoproteins [6]. HPAC cell exhibited polarity (golgi complexes and mitochondria in apical compartment relative to cell nucleus; mucin granules at apical pole), as well as vacuoles, autophagosomes, mitochondria, golgi, endoplasmic reticulum and microvilli (Fig. 1).

Within a short time (1-24 hours), dramatic ultrastructural changes were observed in HPAC cells treated with the TBK1 inhibitor. Although the overall shape of treated HPAC cells remained unchanged from untreated cells, dramatic changes in nuclei morphology were observed. Nuclei were smaller and irregularly shaped with significant invaginations. In the cytoplasm, mucin granules were rarely observed but increasingly large vacuoles and numbers of vacuoles appeared with time of inhibition. Given the reduction in number of mucin granules accompanied by increased number and size of vacuoles from TBK1 inhibition, an underlying compound exocytosis process promoted by TBK1 inhibition was suggested where upon secretion, mucin granule membranes fuse with other mucin granule membranes in the cytoplasm [6]. By 24 hour inhibitor treatment, membrane fusion had quickly progressed to the point where several cells contained essentially 1 large vacuole and mucin granules were rarely found. Autophagosomes persisted at the same frequency as was found in control cells (no statistically significant change in the \# of autophagosomes per cell profile was found with increasing inhibitor treatment time) but the complexity of autophagosomes in cells treated with inhibitor for the longer durations was significantly increased. In summary, TBK1 inhibition in the HPAC cells having aberrant activation of KRAS impacted membrane trafficking and regulation of membrane fusion, cell polarity and autophagic flux. Further studies on the observed increased in complexity of autophagosomes are required to link autophagy to autophagic cell death.

\section{References:}

[1] DA Barbie et al., Nature 462 (2009), p. 108.

[2] VH Cruz and RA Brekken. Journal of Cell Communication and Signaling 12 (2008), p. 83.

[3] LR Gentry et al., Biochimica Biophysica Acta 1843 (2014), p. 2976.

[4] R Van Horn et al., Molecular Cancer Therapy 12 (2013) abstract B229.

[5] LS Yasui and K Owens, International Journal of Radiation Biology 88 (2012), p. 980.

[6] E Kern in “The Pancreas: Biology, Pathobiology, and Disease”, (Raven Press, Ltd., New York).

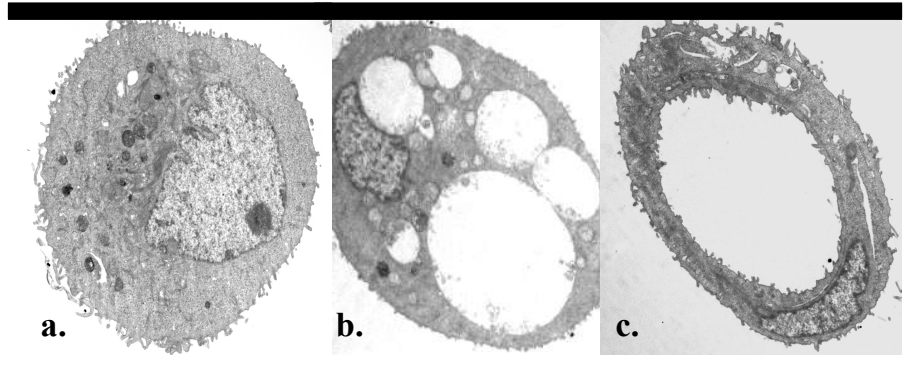

Figure 1. Representative TEM images of HPAC cells not treated with the TBK1 inhibitor (a), treated for 6 hours with inhibitor (b) or treated for 12 hours with the TBK1 inhibitor.

\begin{tabular}{|l|l|l|l|l|l|}
\hline & Controls & $\mathbf{1} \mathbf{~ h r ~ L S N ~}$ & $\mathbf{6} \mathbf{~ h r}$ LSN & $\mathbf{1 2} \mathbf{~ h r ~ L S M}$ & $\mathbf{2 4} \mathbf{~ h r}$ LSN \\
\hline Avg. \# mucin granules/cell +/- SD & $6.1+/-4.3$ & $1.9+/-4.5$ & $0.5+/-0.8$ & $0.9+/-1.8$ & $0.8+/-1.2$ \\
\hline Avg. \# autophagosomes/cell +/- SD & $3.2+/-2.1$ & $4.2+/-4.1$ & $4.2 \%+/-5.5$ & $3.0+/-2.3$ & $8.3+/-6.5$ \\
\hline \% cell profiles exhibiting polarity & $84 \%$ & $42.9 \%$ & $0 \%$ & $22.2 \%$ & $0 \%$ \\
\hline
\end{tabular}

Table 1. Morphometric analysis of autophagosomes and mucin granules in HPAC cells 